\title{
Design and Evaluation of a Connected Insole to Support Healthy Aging of Frail Patients at Home
}

\author{
Yoann Charlon ${ }^{*}$, Antoine Piau ${ }^{2 *}$, Damien Brulin' ${ }^{1}$ Eric Campo ${ }^{1}$ \\ ${ }^{1}$ LAAS-CNRS, Université de Toulouse, CNRS, UT2J, Toulouse, France \\ ${ }^{2}$ Centre Hospitalier Universitaire, Gérontopôle, Toulouse, France \\ Email:ycharlon@gmail.com,piau.a@chu-toulouse.fr,brulin@laas.fr,campo@laas.fr
}

How to cite this paper: Charlon, Y., Piau, A., Brulin, D. and Campo, E. (2019) Design and Evaluation of a Connected Insole to Support Healthy Aging of Frail Patients at Home. Wireless Sensor Network, 11, 67-80. https://doi.org/10.4236/wsn.2019.115005

Received: April 5, 2019

Accepted: May 27, 2019

Published: May 30, 2019

Copyright $\odot 2019$ by author(s) and Scientific Research Publishing Inc. This work is licensed under the Creative Commons Attribution International License (CC BY 4.0).

http://creativecommons.org/licenses/by/4.0/

\begin{abstract}
The main objective of this work is to develop a technological solution to support active aging of frail older individuals. In this perspective, we designed a connected insole in order to encourage frail elderly persons to become more active in their daily life. The project presented in this paper, funded by the French National Research Agency, aims to design and evaluate an original solution to follow and encourage walking activity. The evaluation is performed in two stages: adjusting tests in a living-lab with nine healthy elderly, then clinical evaluation of three frail patients followed over one month. We designed a noninvasive wireless insole, which automatically measures gait parameters and transmits information to a remote terminal via a secure Internet connection. The first laboratory tests of this technological solution showed good reliability measurements as well as good user acceptability. The average distance error obtained on volunteers is $3.2 \%$ and the accuracy of the average walking speed is $96.8 \%$ thanks to design of a calibration system based on a personalized stride length measurement. Finally, the introduction of an induction charging instead of a button cell makes the system perfectly autonomous. In this paper, we present the specifications of the solution, the design of the connected insole, the methods used to measure desired parameters, the results of the living-lab tests, and the work in progress.
\end{abstract}

\section{Keywords}

Connected Object, Smart Insole, Gait Parameters, Frail People, Support Active Aging

\section{Introduction}

The aging population has emerged as a major societal issue. Activity measure-

${ }^{\star}$ These authors contributed equally to this work. 
ment is used as an indicator of elderly health. Recent studies have highlighted frailty as a specific precursor of disability [1]. In France, the care of frail people is performed during hospital visits every three to six months without seamless remote follow-up. Fried et al. criteria [2] are the most commonly used by the medical community to identify and evaluate frail subjects: slow gait speed, decrease in muscle strength, exhaustion, sedentariness, and involuntary weight loss. Several studies showed that walking speed is the most predictive criterion of disability [3] [4]. Frailty is potentially reversible [5] and a number of healthy lifestyle interventions are proposing to increase daily activity of frailty individuals [5] [6] [7].

The use of technology could be relevant for frailty assessment [8], as well as for promoting and monitoring exercise at home [9] [10] and predicting healthrelated events [11]. To encourage sedentary individuals to practice walking, the use of a pedometer can be motivating. Indeed, a review of 2246 publications demonstrates that giving a pedometer and defining aims lead to a daily activity increase of $26.9 \%$ [12]. Thus, proposing a medical follow-up with a connected object could motivate frail people to practice walking.

"Quantified self" devices are already proposed to young robust people to encourage their adherence and motivation (e.g., Fitbit, Nike+). By contrast, in current clinical practice, no devices are used to measure activity and gait speed and give feedback to the patient. In fact, follow-up of frail elderly patients is almost nonexistent. However, to reach our goal we needed to make the system less obtrusive for the end users.

Our overall objective is to develop and validate a smart technological tool to support healthy aging of frail older individuals. Indeed, a connected object would complement the classic follow-up by:

- Motivating the patient thanks to data feedback;

- Providing relevant monitoring data to the physician.

Thanks to end-users and technical requirements we embedded the device into an insoleas:

- An insole is unobtrusive and can be worn without disturbing or stigmatizing the person;

- Several studies showed that inertial sensors worn on the feet alow for accurate measurement of walking speed [13] [14] [15].

We published several papers on the first prototype designs and tests [16] [17] [18] [19]. The first prototype has allowed validation of the technical feasibility of an ambulatory instrument by measuring gait parameters during one month. A first evaluation in living lab and in real life conditions was performed [17] [18] [19]. According to user feedback, it seems that the smart insole could be more easily used than commercial connected pedometers to monitor walking. Moreover, in terms of performance, the smart insole yields better results. The prototype presented here has been iteratively modified as compared to the first version [19], particularly to better fit end-user's needs. The new features are: 
- The design of a calibration system to obtain new ambulatory measures of distance traveled and walking speed with good accuracy (>95\%);

- Use of Bluetooth Low Energy protocol (Bluetooth LE) in order to communicate with a mobile terminal while maintaining good energy autonomy;

- Use of an induction rechargeable LIPO battery for device supply (no need to change the battery);

- Better integration in the insole and use of smoother and thinner materials (reduction to $3 \mathrm{~mm}$ thick).

The primary objective of this work was to evaluate the user interface and the comfort of the new version of the smart insole (acceptability). The paper is organized as follows: Section 2 describes project features; Section 3 focuses on design of the final prototype and on the method used to measure gait parameters; Section 4 presents user interfaces; Section 5 concerns evaluation in living-lab; Section 6 presents the conclusion and work in progress.

\section{Project Features}

\subsection{Medical specifications}

Currently, Fried criteria associated with walking are measured at the hospital by standard tests (e.g. four meters walking in a straight line and use of a stopwatch). The smart insole must provide these indicators to the physician at home over time. According to the medical stakeholder's needs, the insole should be able to measure according to time (day, week, and month):

- Distance covered and the number of steps (only one insole is instrumented, we measure the number of strides and we multiply by 2 to have the number of steps);

- Activity periods;

- Average gait speed during activity periods.

To be relevant, gait speed accuracy must be greater than 95\% during natural gait. To increase accuracy, we propose to calibrate the smart insole on the real gait of the individual. The system must inform:

- The end user about his activity to motivate him to walk;

- The physician who sets objectives for this patient and follows his/her evolution.

The main constraints related to obtrusiveness are the following:

- The device should need no maintenance over three months (time between two medical consultations);

- The system must be autonomous for measurements and communication.

\subsection{Operational Setup of the Monitoring System}

The operational setup of the system consists of (Figure 1):

- A touch pad to collect data from the insole;

- A secure remote database;

- A web application for the patient and the physician. 


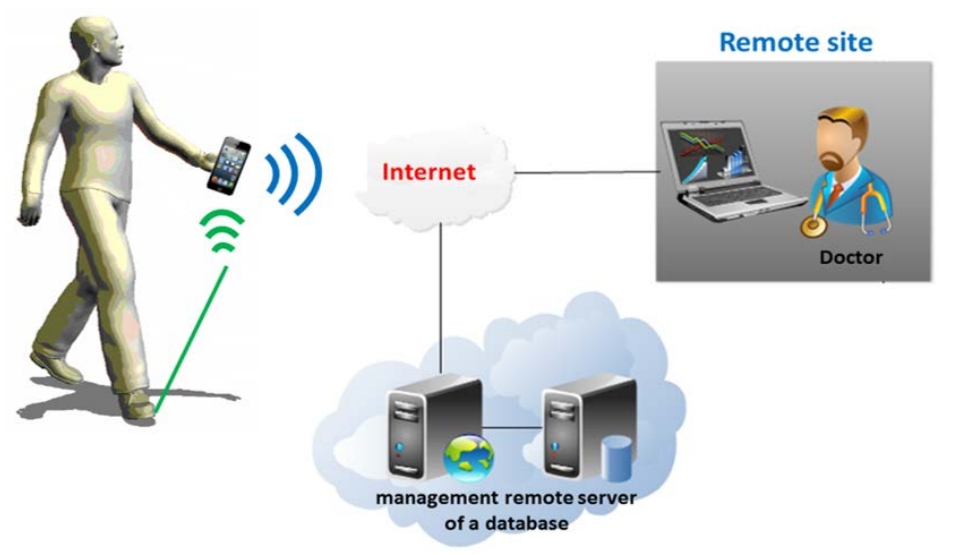

Figure 1. Operational setup.

\section{Material and Method}

\subsection{Design of the Final Prototype}

Discussions with professionals in podiatry and initial integrations tests [16] [17] led us to apply the following constraints: the system should be placed under the arch of the foot to maintain comfort of the insole; maximum dimensions of the module must be $50^{\star} 40^{\star} 2 \mathrm{~mm}$.

The device integrated in the insole (Figure 2) included: a System on Chip (NRF51822) which is composed of a $2.4 \mathrm{Ghz}$ transceiver and a microprocessor (Cortex-M0), a 3 axis accelerometer (ADXL362), and a wireless power receiver ( $\mathrm{Rx}$ Coil) associated with a battery charge controller (bq51050B). The power supply is performed with a rechargeable LIPO battery (100 mAh).

The induction charger (Figure 3) is an induction charger unit (EP-PG900I) integrated in a heel-piece in order to directly charge the insole inside the shoe. The dimensions of the device are $45^{\star} 40^{\star} 2 \mathrm{~mm}$ for a total weight of $10 \mathrm{~g}$, including the battery and Rx Coil.

The insole is not intended to measure balance or stabilometry but active walking and average walking speed. Instrumenting the two insoles is therefore of no interest in this case.

For the three healthy elderly persons followed up in their daily life at home over one month, the material on the following figure has been distributed.

The RESPECT instrumented insole pair replaces the original insole pair as described in Figure 4.

To charge the instrumented insole, the heel-piece must be connected as shown in Figure 5. When the instrumented insole is charged the light turns green.

Several people can use the tablet: the person followed, the life partner, or a helper. The tablet aims to coach the activity but its use is not mandatory. In this case, the system measures the gait parameters and remotely transfers the data without direct feedback to the user. The main objective is to set up a medical follow-up. 


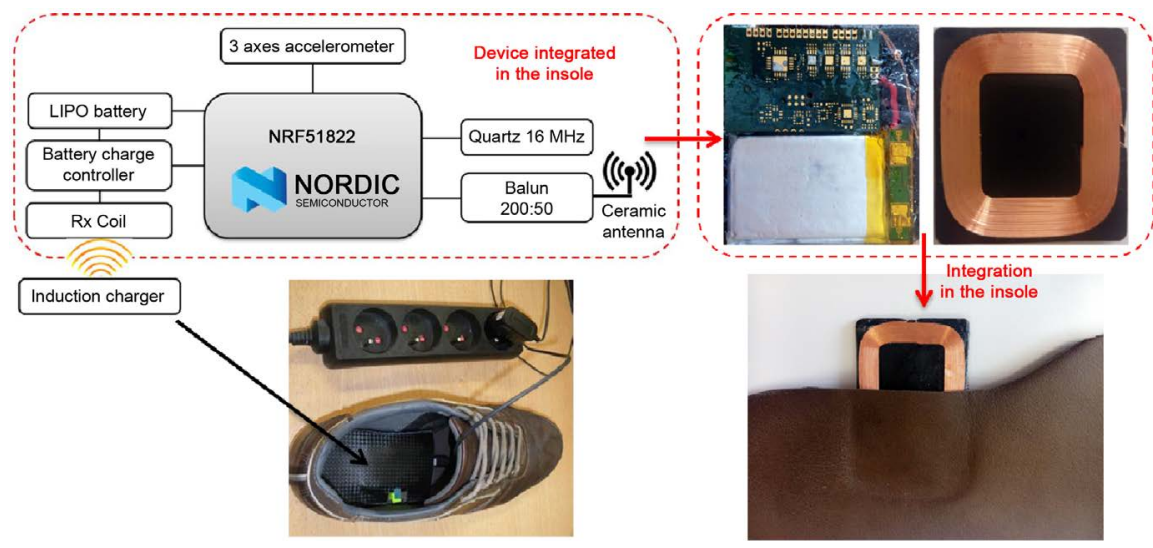

Figure 2. Connected insole and its induction charger.

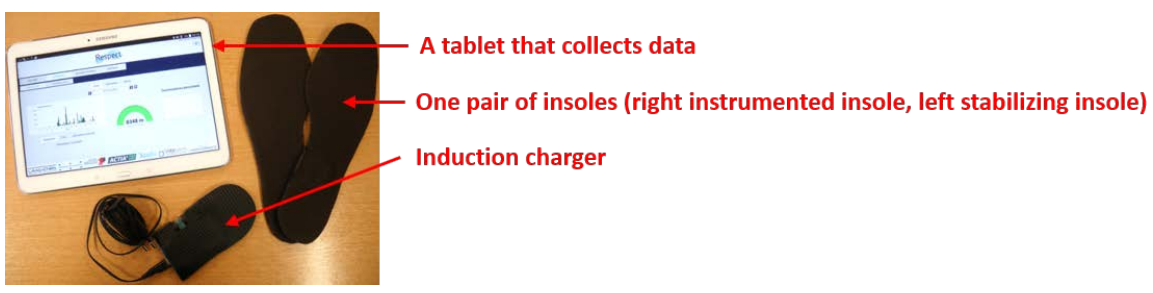

Figure 3. Material distributed at each volunteer followed at home.

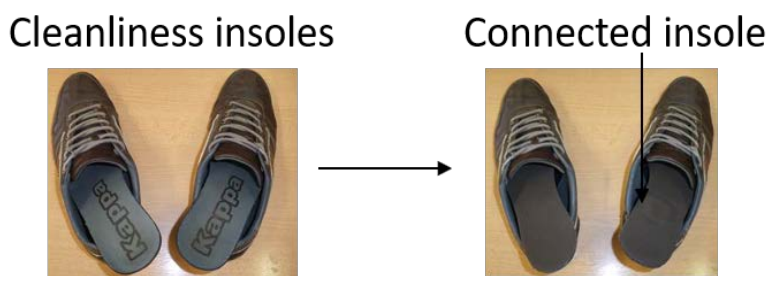

Figure 4. Cleanliness insoles replaced by RESPECT insoles.
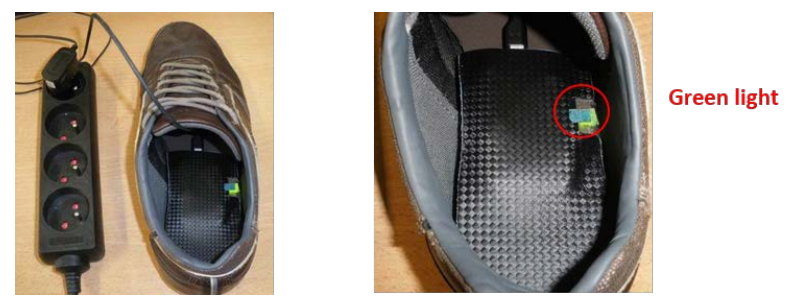

Figure 5. Charge of the instrumented insole.

The first version of the insole was communicating with ZigBee and we needed a receiver connected to a computer [17]. Now, with Bluetooth communication, the smart insole can communicate directly with smartphones and tablets. The first version of the smart sole has 67 days of battery life with a button battery [17]. A change of the smart insole is required during the 3 months of follow-up because the button cell was fully integrated and cannot be replaced. With the LIPO rechargeable battery, only one smart insole is needed during the entire follow-up period. The user must charge the insole once a week, which seems acceptable. 


\subsection{Method for Measuring the Gait Parameters}

The main objective is to provide activity data to the physician and to the patient (distance and number of steps, active minutes) and a reliable speed indicator to the physician (less than 5\% error with respect to real walking speed). In literature, gait speed calculation is based on stride length measurement [13] [14] [15] [20] [21]. Two methods are mainly used from inertial sensors placed on shoes to calculate stride length:

- Mathematical integration of inertial signals [13] [14] [15];

- Analytical method [20] [21].

Mathematical integration is attractive in terms of measurement accuracy with a margin of error that may be close to $1 \%$. However, that method requires the combination of an accelerometer and a gyrometer which induce high-energy consumption. The analytical method is less accurate with a margin of error of about 5\%. The major drawback lies in the need to calibrate the system on the gait of the user. However, this method seems to be an interesting alternative in order to reduce the system's energy consumption because only a three-axis accelerometer is needed. Thus, the development of an analytical method is more appropriate to our project features to reduce energy consumption while still offering about $95 \%$ accuracy.

The first version of our method was presented in [17]. Here we present method details and the latest improvements. The method for measuring the average gait speed is composed of three levels:

- Level 1: strides detection and measurement of the average cadence;

- Level 2: calibration using a specifically designed system;

- Level 3: computation of the average stride length, average gait speed and distance covered for each gait period.

The method for strides detection (Level 1) remains unchanged and is described elsewhere [19]. Stride detection is realized as follow (Figure 6): Compute acceleration magnitude, mean acceleration, and acceleration variance and stride detection by using thresholds on the acceleration variance and time limits. After stride detection, walking cadence is computed by using a sliding window on the last three strides. Errors observed with this method were less than $1 \%$ of the number of strides [19].

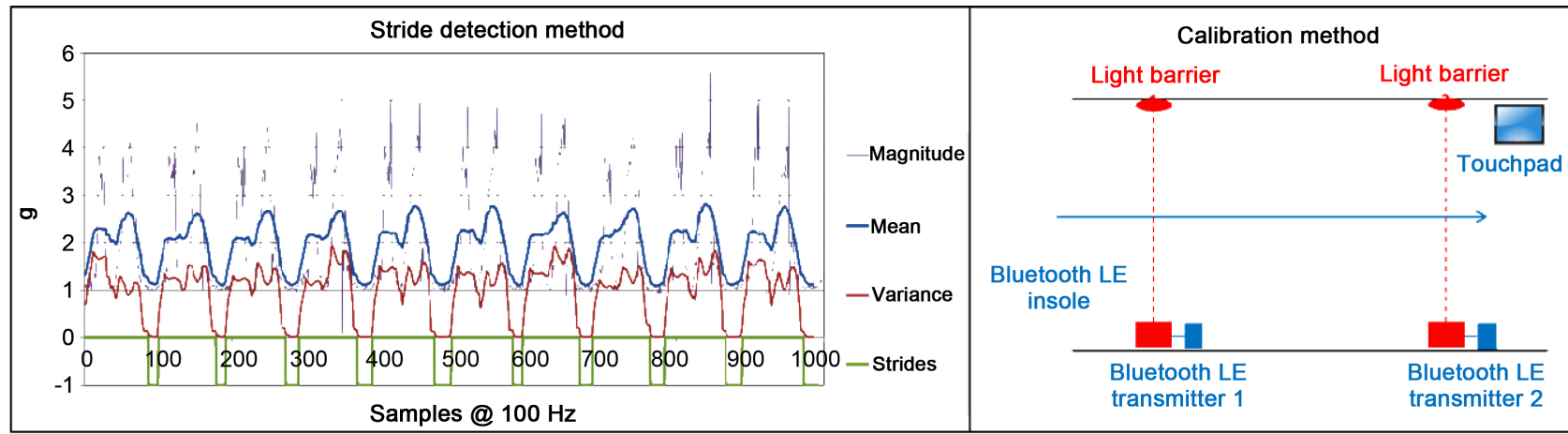

Figure 6. Stride detection and calibration methods (Level 1 and 2). 
Calibration (Level 2) is modified and simplified as compared to the previous model [16] since the patient performs only one walk. Moreover, the calibration system now communicates in Bluetooth Low Energy. During calibration, two parameters are measured: average gait speed and average cadence while the user walks naturally at a medium speed for four meters (as asked in medical procedure). The calibration system is composed of the following elements (Figure 6):

- An instrumented insole that measures the average cadence;

- A double optical barrier that measures the average speed on four meters;

- A touchpad that collects the data and computes the stride length (Equation (1)).

Calibration must be performed during a stabilized walk. Thus, the user starts one meter before the first optical barrier and stops one meter after the second optical barrier. In this way, gait speed is constant between the two optical barriers (Figure 7).

The smart insole measures the cadence after the second stride when the walk is stabilized. Calibration software installed on the touchpad collects the average gait speed and average cadence in order to determine the stride length (Equation (1)).

Computation of the average stride length has evolved comparing with previous work [17]; now the following equation is used:

$$
\text { stride }=G_{s} / C
$$

where:

- Stride is the average stride length in meters as computed by the calibration system;

- $G_{s}$ is the average gait speed in meters per second as determined by the calibration system;

- $C$ is the average cadence in Hertz as determined by the calibration system.

After calibration, the connected insole computes the average gait speed $\left(G_{w}\right)$ for each continuous walk using the following equation:

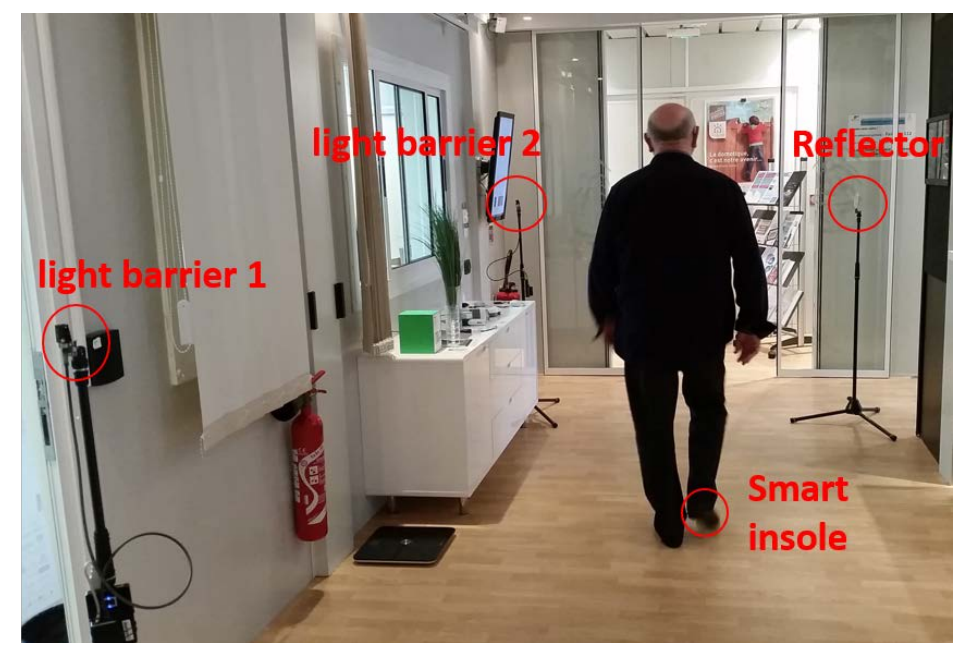

Figure 7. Calibration system with a volunteer. 


$$
G_{w}=C * \text { stride }
$$

where:

- $G_{W}$ is the average gait speed in meters per second as determined by the smart insole;

- $C$ is the average cadence in Hertz as determined by the smart insole;

- Stride is the average stride length in meters as determined by the calibration system.

In order to evaluate the accuracy of our average gait speed computing method, three volunteers conducted tests using three different speeds (slow, medium and fast). The smart insole was calibrated to fit the stride length of each volunteer. Distance covered is computed by adding successive stride lengths. The mean error obtained over a distance of $400 \mathrm{~m}$ (athletics stadium) for our three volunteers is shown in Table 1.

The mean distance error obtained on 400 meters (athletics stadium) for our three volunteers is $3.2 \%$.

The speed error is the same because the real time clock of the smart insole is accurate to the millisecond (speed = distance/time). $96.8 \%$ accuracy on average walking speed meets our specifications (greater than the 95\% accuracy expected).

\section{User Interfaces}

The end-user's interfaces (physician and patient) are touchpad Web applications, which allow an interactive and user-friendly follow up. The personal objectives set by the physician were integrated into the dashboard of the patient's interface (motivational coaching). Encouragement or congratulatory messages also are displayed depending on the fulfillment of the objectives. These interfaces monitor the number of steps, the distance covered, active minutes (continuous walking during at least five minutes) and the mean gait speed (only on the physician interface). This feedback is given to the user for daily use at home. A screen capture of the patient interface is shown in Figure 8. This will enable the user to be kept informed of his/her progress with respect to individual walking objectives, the evolution of his/her personal health status and any alert in the event that an abnormal trend in indicators occurs. The physician interface is presented in Figure 8.

Table 1. Mean error of distance covered.

\begin{tabular}{cccc}
\hline & Volunteer 1 & Volunteer 2 & Volunteer 3 \\
\hline Slow speed & $3.9 \%$ & $4.2 \%$ & $3.6 \%$ \\
Medium speed & $2.6 \%$ & $2.5 \%$ & $2.4 \%$ \\
Fast speed & $3.2 \%$ & $3.2 \%$ & $3.3 \%$ \\
Mean error & $3.2 \%$ & $3.3 \%$ & $3.1 \%$ \\
\hline
\end{tabular}




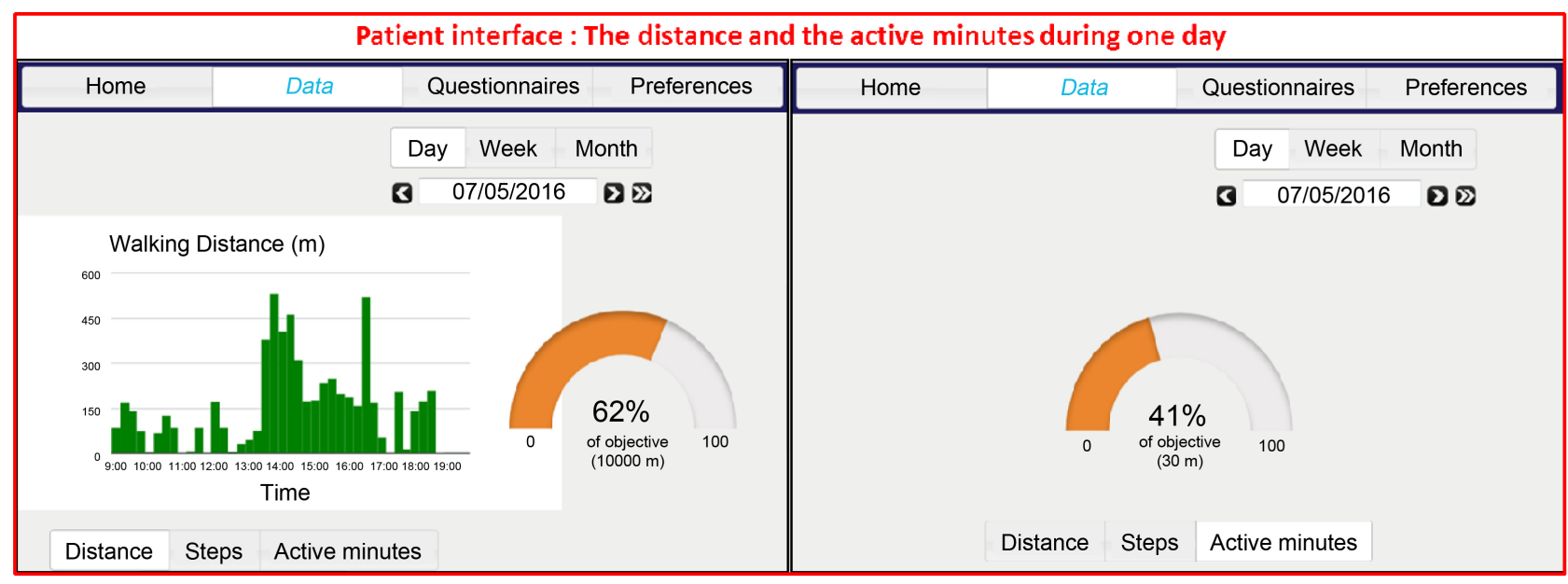

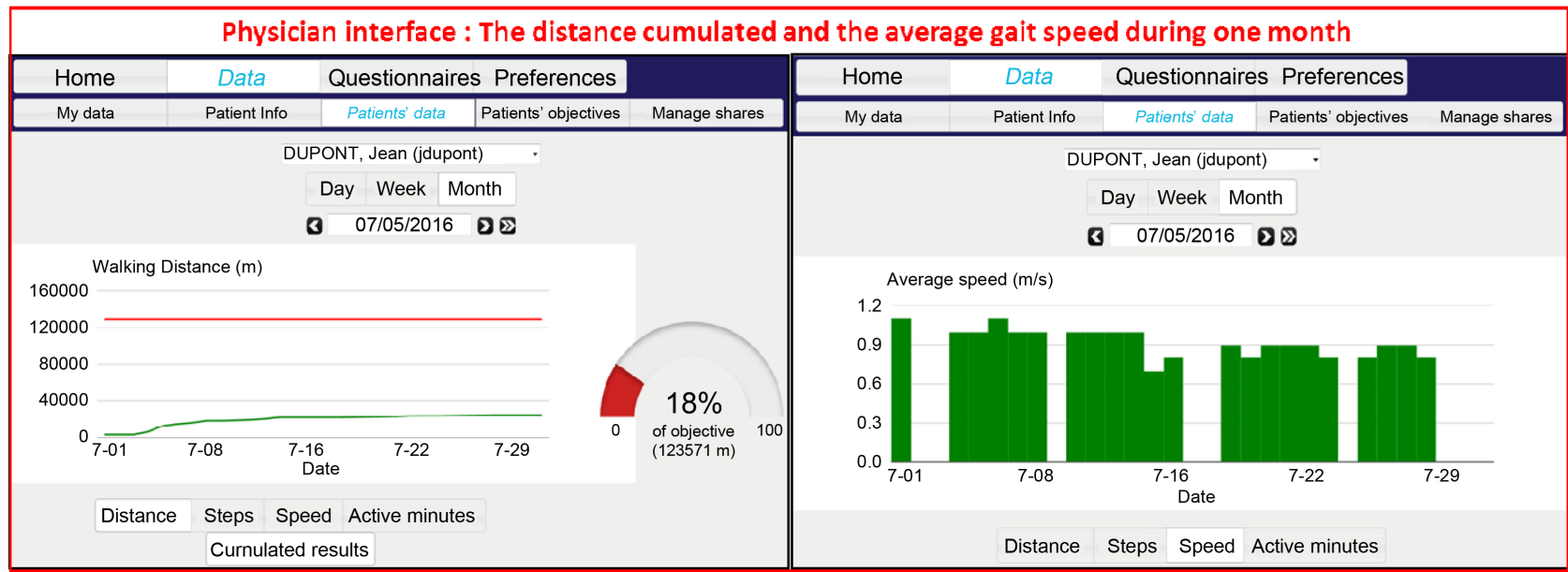

Figure 8. User interfaces.

\section{Living-Lab Results}

First, the connected insole was worn continuously by a young volunteer during one month, without discomfort noted by him and without damage to the module. The energy autonomy of the connected insole with a fully charged battery is four days. Then, the smart insole was evaluated in two phases: first, tests in a living-lab with nine healthy elderly persons, then a clinical evaluation with thirty frail elderly persons followed in real life setting over three months. The living-lab evaluation is currently complete and the field clinical evaluation is in progress. In this section, we present the objectives, outcomes and results of the living-lab evaluation. The main objectives were:

- Insole: test the lifetime of the electronic circuit, test the new induction charging system, check and improve the overall functioning (e.g. data transmission) before the clinical phase;

- Calibration: test the system and the protocol;

- Measures: test the accuracy of the gait parameters measurement (distance and speed);

- Charging system: the must be easy to use 
- Comfort: check the comfort of the smart insole for the end user;

- Web interface: gather user feedback.

Living-lab tests were carried out in two sub-phases:

1) Living-lab phase 1: evaluation by nine healthy elderly persons of the calibration protocol and of the smart insole during one half-hour on a defined path.

2) Living-lab phase 2: three healthy elderly persons followed up over one month in their daily lives.

The collection of user opinions concerning the use of the sole, the interface, the charging system, but also concerning the calibration protocol (e.g. comfort of the sole, ergonomics of the mobile application) is carried out using semi-directive interviews with the user. The data collection is conducted by a clinical research assistant.

\subsection{Participants}

A total of nine patients were enrolled. Among them, there were six women (66.6\%) and three men. The mean age was 70.1 years (65 to 75$)$. All were living at home. None were suffering from dementia or mild cognitive impairment. They did not present any functional disability, and only one volunteer suffered from urinary incontinence.

This is a multiphase study, with a participatory approach that allows iterative modifications of the prototype between each phase. In line with this method, we are testing the insole in increasingly older and more frail populations. The first phases concerned young and non-pathological users, while the current field evaluation is carried out in older people with multiple diseases.

\subsection{Living-Lab Phase 1, Results}

The experiment was set up in the smart home of the University Institute of Technology in Blagnac, France (MIB) [19]. This smart home is a technological, scientific and human platform. In the standard configuration, this flat of $70 \mathrm{~m}^{2}$ is equipped with a networking infrastructure adaptable to a valid, a frail or a disabled person (Figure 1). This home is equipped with various kinds of sensors and technical aids. The nine volunteers used the calibration system and the smart insole for one half-hour on a defined path. Results of phase 1 of the living-lab are:

- Insole: judged comfortable by all participants;

- Calibration: functional but the protocol is too complicated for the elderly users who should accomplish two passages (see detail [16]);

- Measures: the average speed and distance covered as measured on the defined path are very close to the speeds measured during the calibration phase (difference $<5 \%$ for each volunteer);

- Charging system, comfort, web interface: not tested in Phase 1.

\subsection{Living-Lab Phase 2}

Three of the nine healthy elderly persons were followed up in their daily life at 
home over one month. Results of Phase 2 of the living-lab are:

- Insole: one insole circuit was broken after three weeks of use; one bug detected during the transmission of data (synchronization after several days of use); smart insole autonomy is acceptable;

- Calibration: the simplified protocol with only one passage is validated, but the calibration system is too heavy to be used easily at home;

- Measures: follow-up of gait parameters in patients' daily lives implies a variation in walking conditions (stairs, falls, weather, shuffling at home, etc.). To analyze the data, we asked users to note their activities in a log book. Over the months that followed, one volunteer completed a hike of $8.2 \mathrm{~km}$ (marked path). The distance calculated by the connected insole was $8.6 \mathrm{~km}$ (a difference of about $5 \%$ ). We noted that average speed varies from day to day. Indeed, short trips, especially at home, are unstable (shuffling). It is obvious that the daily speed indicator should be calculated using stabilized walks. Several treatments were carried out and compared. Following these treatments, it appears that the use of the three longest walking periods each day makes it possible to obtain reliable indicators (with a variation of less than $5 \%)$;

- Charging system: considered easy to use;

- Comfort: the smart insole was declared comfortable and quickly forgotten by each user;

- Web interface: some texts and curves are too small and several colors are not appropriate.

Improvements made after the second phase of the living-lab are:

- Insole: circuit was varnished and encapsulated in epoxy glue to protect it from humidity and shocks, before integration into the insole with a neoprene adhesive to prevent chafing; The bug is fixed;

- Measures: use of three longer walking periods to compute average gait speed per day;

- Calibration: we used a touchpad instead of a computer to collect the data (Figure 6 and Figure 7)-optical barrier supports are lighter and a carrying case also was used;

- Web Interface: improved ergonomics based on end-user's feedback (Figure 8).

The improvements made at the end of Phase 2 of the living-lab were laboratory tested. They are currently being validated in the clinical phase.

\section{Discussion}

Our purpose was to design a technological tool to support continuous follow-up with minimal invasiveness both at home and in the outside environment. Potentially, this could be helpful to promote healthy lifestyle recommendations for a frail older population. In this perspective, we propose a connected insole to support active aging. In this paper, we have presented a new prototype of our 
connected insole to better suit end-users' needs. These improvements are:

- Use of Bluetooth Low Energy protocol (Bluetooth LE) in order to communicate with a mobile terminal while maintaining good energy autonomy;

- Device supply by a rechargeable LIPO battery by induction.

The living-lab stage with nine elderly volunteers took place. This test stage was successful and highlighted the technical and ergonomic improvements desirable before deploying Version 2 of our solution in 30 patients' homes, specifically:

- Circuit protection to increase its lifetime;

- Improved patient interface by better presentation of indicators and graphics;

- Improved data collection;

- Adaptation of the calibration system for mobile use at home.

\section{Conclusion}

End users' interfaces have been considered to be ergonomic. Evaluation of the acceptability of the insole is very encouraging. To date, we do not have objective clinical data on the use of instrumented insoles beyond a few hours of laboratory testing in this specific population of pre-frail and frail older adults. Our results will help health care researchers and technical stakeholders to establish specifications for comparable devices. This work opens up an interesting prospect for the implementation of continuous follow-up at home. It now is necessary, however, to evaluate this solution with frail elderly people. A clinical field trial is in progress and expected to allow a first evaluation of the ecosystem (users, physician and service providers), as well as an evaluation of the implementation process.

\section{Acknowledgements}

The authors thank the French National Research Agency and CNSA for financial support through the TECSAN program (ANR-13-TECS-0007-2013-RESPECT Project) and the ACTIA Company that developed the user interface and provided assistance during the living lab tests.

\section{Conflicts of Interest}

The authors declare that there is no conflict of interest.

\section{References}

[1] Van Kan, G.A., Rolland, Y., Bergman, H., Morley, J.E., Kritchevsky, S.B. and Vellas, B. (2008) The I.A.N.A. Task Force on Frailty Assessment of Older People in Clinical Practice. The Journal of Nutrition Health and Aging, 12, 29-37. https://doi.org/10.1007/BF02982161

[2] Fried, L.P., Ferrucci, L., Darer, J., Williamson, J.D. and Anderson, G. (2004) Untangling the Concepts of Disability, Frailty, and Comorbidity: Implications for Improved Targeting and Care. The Journals of Gerontology: Series A, 59, 255-263. https://doi.org/10.1093/gerona/59.3.M255 
[3] Cesari, M., Kritchevsky, S.B., Penninx, B.W.H.J., Nicklas, B.J., Simonsick, E.M., Newman, A.B., Tylavsky, F.A., Brach, J.S., Satterfield, S., Bauer, D.C., Visser, M., Rubin, S.M., Harris, T.B. and Pahor, M. (2005) Prognostic Value of Usual Gait Speed in Well-Functioning Older People-Results from the Health, Aging and Body Composition Study. Journal of the American Geriatrics Society, 53, 1675-168. https://doi.org/10.1111/j.1532-5415.2005.53501.x

[4] Montero-Odasso, M., Schapira, M., Soriano, E.R., Varela, M., Kaplan, R., Camera, L.A. and Mayorga, L.M. (2005) Gait Velocity as a Single Predictor of Adverse Events in Healthy Seniors Aged 75 Years and Older. The Journals of Gerontology: Series A, 60, 1304-1309. https://doi.org/10.1093/gerona/60.10.1304

[5] Pahor, M., Blair, S.N., Espeland, M., Fielding, R., Gill, T.M., Guralnik, J.M., Hadley, E.C., King, A.C., Kritchevsky, S.B., Maraldi, C., Miller, M.E., Newman, A.B., Rejeski, W.J., Romashkan, S. and Studenski, S. (2006) Effects of a Physical Activity Intervention on Measures of Physical Performance: Results of the Lifestyle Interventions and Independence for Elders Pilot (LIFE-P) Study. The Journals of Gerontology: Series A, 61, 1157-1165. https://doi.org/10.1093/gerona/61.11.1157

[6] Nelson, M.E., Layne, J.E., Bernstein, M.J., Nuernberger, A., Castaneda, C., Kaliton, D., Hausdorff, J., Judge, J.O., Buchner, D.M., Roubenoff, R. and Fiatarone Singh, M.A. (2004) The Effects of Multidimensional Home-Based Exercise on Functional Performance in Elderly People. The Journals of Gerontology: Series A, 59, 154-160. https://doi.org/10.1093/gerona/59.2.M154

[7] Daniels, R., van Rossum, E., de Witte, L., Kempen, G.I. and van den Heuvel, W. (2008) Interventions to Prevent Disability in Frail Community-Dwelling Elderly: A Systematic Review. BMC Health Services Research, 8, 278.

https://doi.org/10.1186/1472-6963-8-278

[8] Fontecha, J., Hervás, R., Bravo, J. and Navarro, F.J. (2013) A mobile and Ubiquitous Approach for Supporting Frailty Assessment in Elderly People. Journal of Medical Internet Research, 15, e197. https://doi.org/10.2196/jmir.2529

[9] Makai, P., Perry, M., Robben, S.H., Schers, H.J., Heinen, M.M., Olde Rikkert, M.G., et al. (2014) Evaluation of an eHealth Intervention in Chronic Care for Frail Older People: Why Adherence Is the First Target. Journal of Medical Internet Research, 16, e156. https://doi.org/10.2196/jmir.3057

[10] Yamada, M., Mori, S. and Nishiguchi, S. (2012) Pedometer-Based Behavioral Change Program Can Improve Dependency in Sedentary Older Adults: A Randomized Controlled Trial. The Journals of Gerontology: Series A, 66A, 1108-1113. https://doi.org/10.1093/gerona/glr101

[11] Pajala, S., Era, P., Koskenvuo, M., Kaprio, J., Törmäkangas, T. and Rantanen, T. (2008) Force Platform Balance Measures as Predictors of Indoor and Outdoor Falls in Community-Dwelling Women Aged 63-76 Years. The Journals of Gerontology. Series $A, 63,171-178$. https://doi.org/10.1093/gerona/63.2.171

[12] Bravata, D.M., Smith-Spangler, C., Sundaram, V., Gienger, A.L., Lin, N., Lewis, R., Stave, C.D., Olkin, I. and Sirard, J.R. (2007) Using Pedometers to Increase Physical Activity and Improve Health: A Systematic Review. The Journal of the American Medical Association, 298, 2296-2304. https://doi.org/10.1001/jama.298.19.2296

[13] Mariani, B., Hoskovec, C., Rochat, S., Büla, C., Penders, J. and Aminian, K. (2010) 3D Gait Assessment in Young and Elderly Subjects Using Foot-Worn Inertial Sensors. Journal of Biomechanics, 43, 2999-3006. https://doi.org/10.1016/j.jbiomech.2010.07.003

[14] Martin Schepers, H., van Asseldonk, E.H.F., Baten, C.T.M. and Veltink, P.H. (2010) Ambulatory Estimation of Foot Placement during Walking Using Inertial Sensors. 
Journal of Biomechanics, 43, 3138-3143.

https://doi.org/10.1016/j.jbiomech.2010.07.039

[15] Tien, I., Glaser, S.D., Bajcsy, R., Goodin, D.S. and Aminoff, M.J. (2010) Results of Using a Wireless Inertial Measuring System to Quantify Gait Motions in Control Subjects. IEEE Transactions on Information Technology in Biomedicine, 14, 904-915. https://doi.org/10.1109/TITB.2009.2021650

[16] Charlon, Y., Bettahar, F. and Campo, E. (2013) Design of a Smart Shoe Insole to Monitor Frail Older People. International Conference on Modern Well-Being for Societies and Territories, 1-6.

[17] Charlon, Y., Campo, E., Brulin, D., Bettahar, F. and Piau, A. (2015) Smart Insole for Measuring Actimetry of Frail People. Med-e-Tel 2015, 1-7.

[18] Charlon, Y. (2014) Conception de dispositifs électroniques portés pour le suivi de l'état de santé des personnes âgées. 1-216.

[19] Piau, A., Charlon, Y., Campo, E., Vellas, B. and Nourhashemi, F. (2015) A Smart Insole to Promote Healthy Aging for Frail Elderly Individuals: Specifications, Design, and Preliminary Results. JMIR Rehabilitation and Assistive Technologies, 2, e5. https://doi.org/10.2196/rehab.4084

[20] Ladetto, Q. (2000) On Foot Navigation: Continuous Step Calibration Using Both Complementary Recursive Prediction and Adaptive Kalman Filtering. 13th International Technical Meeting of the Satellite Division of the Institute of Navigation, Salt Lake City, UT, 19-22 September 2000, 1735-1740.

[21] Shin, S.H., Park, C.G., Kim, J.W., Hong, H.S. and Lee, J.M. (2007) Adaptive Step Length Estimation Algorithm Using Low-Cost MEMS Inertial Sensors. 2007 IEEE Sensors Applications Symposium, San Diego, CA, 6-8 February 2007, 1-5. https://doi.org/10.1109/SAS.2007.374406 